\title{
Self-construal and the processing of covariation information in causal reasoning
}

\author{
KYUNGIL KIM \\ Ajou University, Suwon, Korea \\ AND \\ Lisa R. Grimm AND ARThur B. MARKMAN \\ University of Texas, Austin, Texas
}

\begin{abstract}
Causal induction provides a nice test domain for examining the influence of individual-difference factors on cognition. The phenomena of both conditionalization and discounting reflect attention to multiple potential causes when people infer what caused an effect. We explored the hypothesis that individuals with an independent self-construal are relatively less sensitive to context (other causes) than are individuals with an interdependent self-construal in this domain. We found greater levels of conditionalization and data consistent with discounting for participants in whom we primed an interdependent self-construal than for participants in whom we primed an independent self-construal.
\end{abstract}

Research on cultural differences and expertise has highlighted the presence of significant individual differences in performance on cognitive tasks that have often been thought to represent more universal cognitive tendencies (Lopez, Atran, Coley, Medin, \& Smith, 1997; Proffitt, Coley, \& Medin, 2000). For example, the research of Nisbett and his colleagues showed that members of East Asian cultures were more likely to prefer proverbs that embody contradictions than were members of Western cultures (Peng \& Nisbett, 1999).

A potential difficulty with research on intact populations is that it can be difficult to isolate the underlying variables that lead to these differences in performance (Hong \& Chiu, 2001). Furthermore, because of the kinds of tasks typically studied in the research on cultural differences, it can be difficult to isolate the aspects of performance that change.

We addressed this issue by exploring the role of a particular individual-difference variable - self-construalthat has been demonstrated to differ between cultures and has been shown to lead to differences in cognitive performance. We examined the influence of differences in selfconstrual on causal induction from observed events. This domain is useful because we can provide a quantitative assessment of the degree to which people attend to contextual information and, consequently, can test a specific hypothesis about the nature of individual differences.

We will begin with a brief description of self-construal as an individual-difference variable. Then, we will examine how causal induction can be used to assess people's attention to context. Finally, we will present an experiment in which the influence of differences in self-construal on causal induction was explored.

\section{Self-Construal and Cognition}

Self-construal is defined as an individual's mental representation of himself or herself (Markus \& Kitayama, 1991). A person's self-construal is the information an individual uses to answer to the question, "Who am I"? For example, a woman might represent herself as a mother, a psychologist, a lover of jazz, or a person who dislikes anchovies.

One systematic difference in self-construals across individuals is the degree to which they are interdependent versus independent (Markus \& Kitayama, 1991). Interdependent self-construals contain descriptors that relate the self to others explicitly (e.g., the descriptor mother links the individual to her children). Independent self-construals contain descriptors that are not connected to others (e.g., anchovy-lover does not implicate other people).

Three aspects of research on self-construal are relevant to this article. First, there is evidence for significant group differences in self-construal. All else being equal, women and members of Eastern cultures have more interdependent descriptors and fewer independent descriptors in their selfconstruals than do men (Cross \& Madson, 1997) and members of Western cultures (Markus \& Kitayama, 1991).

Second, groups with an interdependent self-construal tend to be more sensitive to contextual or relational information in the environment than do those with an independent self-construal. For example, Eastern participants rely on relational information more than do Western participants in tasks involving attention and memory (Masuda \& Nisbett, 2001), perception (Ji, Peng, \& Nisbett, 2000), categorization (Ji, Zhang, \& Nisbett, 2004), and reasoning (Choi, Nisbett, \& Norenzayan, 1999). 


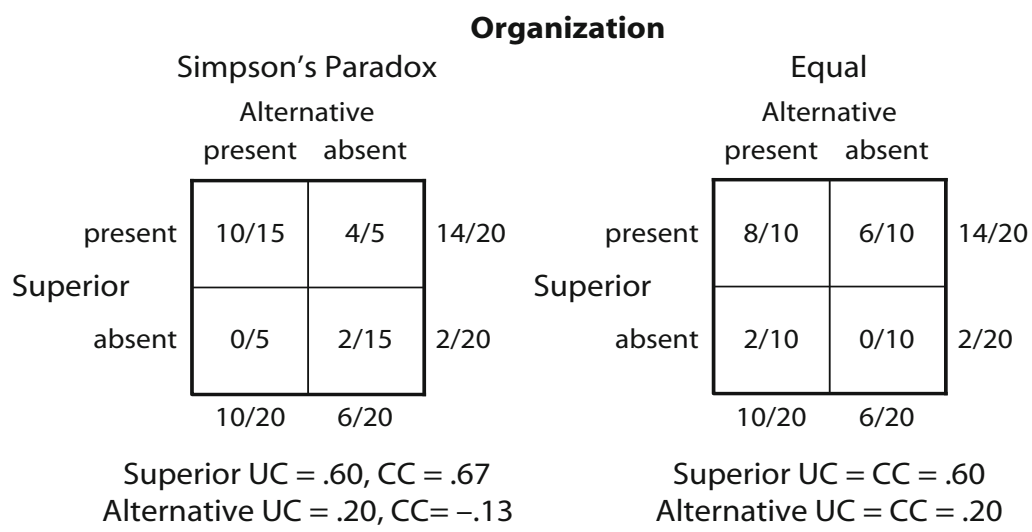

Figure 1. Cell information and the unconditional contingencies (UCs) and conditional contingencies (CCs) of the causes (i.e., liquids) for the Simpson's paradox organization and equal organization used in the study (Spellman, Price, \& Logan, 2001, Figure 2).

Third, self-construal can be manipulated in the laboratory by priming subjects with interdependence or independence, using a pronoun-circling task: first-person plural pronouns (e.g., we and our) for interdependence and first-person singular pronouns (e.g., $I$ and mine) for independence (Gardner, Gabriel, \& Lee, 1999; Trafimow, Triandis, \& Goto, 1991). In Gardner et al., participants primed with interdependence endorsed Eastern collectivist values more than Western individualist values, whereas those primed with independence showed the opposite pattern.

Priming studies have linked differences in self-construal to differences in people's propensity to process relational information (Constantine, 2001; Haberstroh, Oyserman, Schwarz, Kuhnen, \& Ji, 2002; Kim \& Markman, 2006; Kuhnen, Hannover, \& Schubert, 2001; Kuhnen \& Oyserman, 2002; van Baaren, Maddux, Chartrand, de Bouter, \& van Knippenberg, 2003). For example, Kuhnen et al. demonstrated that those with an independent self-construal performed better on an embedded figures task, as compared with those primed with interdependence.

A potential limitation of this previous work is that the concept of contextual or relational information is based on the shared intuition of researchers. In this article, we turn to the domain of causal reasoning, because previous research has already developed a rigorous definition and related empirical work on the role of contextual information in causal judgment.

\section{Causal Reasoning and Contextual Information}

People are able to make judgments about whether a candidate cause leads to an effect by examining the degree to which the cause and the effect covary. Intuitively, if a candidate cause, C, reliably leads to an effect, E, E should occur more often when $\mathrm{C}$ occurs than when it does not. If $\mathrm{C}$ reliably inhibits $\mathrm{E}, \mathrm{E}$ should occur less often when $\mathrm{C}$ occurs than when it does not. Finally, if $\mathrm{C}$ does not influence $\mathrm{E}$, the likelihood that $\mathrm{E}$ will occur should be about the same regardless of whether $\mathrm{C}$ occurs. This intuition is captured mathematically in the $\Delta P$ rule:

$$
\Delta P=P(\mathrm{E} \mid \mathrm{C})-P(\mathrm{E} \mid \sim \mathrm{C}),
$$

where $\mathrm{C}$ is a candidate cause and $\mathrm{E}$ is an effect (Wasserman, Chatlosh, \& Neunaber, 1983). When $\Delta P$ is positive, the cause reliably leads to the effect; when $\Delta P$ is negative, the cause reliably inhibits the effect; and when $\Delta P$ is 0 , the cause and the effect are unrelated. This formulation of the $\Delta P$ rule is called an unconditional contingency (UC) between the cause and the effect, because no other potential causes are considered.

In most real-world situations, of course, there are many possible candidate causes for an effect. If those candidate causes often appear together, they are statistically confounded, and so unconditional contingencies do not give a reliable estimate of causality. To address this concern, Melz, Cheng, Holyoak, and Waldmann (1993) and Cheng (1993) proposed that reasoners consider conditional contingencies (CCs), which are determined in the context of alternative causes. Conditional contingencies take into account the presence or absence of alternative causes when the influence of a cause on an effect is calculated (Spellman, 1996a, 1996b; Spellman, Price, \& Logan, 2001). ${ }^{1}$ For example, the conditional $\Delta P$ rule,

$$
\Delta P_{A \mid B}=P(\mathrm{E} \mid \mathrm{A} \text { and } \mathrm{B})-P(\mathrm{E} \mid \sim \mathrm{A} \text { and } \mathrm{B}) \text {, }
$$

incorporates the probability that the effect occurred given the presence of both causes A and B and the probability that the effect occurred given the absence of $\mathrm{A}$ and the presence of B.

Spellman (1996a, 1996b) demonstrated that people are capable of considering this conditional contingency by creating an environment in which attending to the unconditional contingencies and the conditional contingencies between causes and effects would lead to different assessments of the effectiveness of the causes. Figure 1, which illustrates a design used by Spellman to address this issue, depicts contingency tables for two pairs of causes that might lead to an effect. In Spellman's (1996a, 1996b) studies (and in the experiment we performed here), the candidate causes were liquids that might act as fertilizers for plants, 
and the effect was whether a given plant bloomed. People were exposed to events in which a particular combination of fertilizers was presented. Each cell of the contingency table shows the number of times the plant blooms given this combination out of the total number of presentations of that combination over the course of the experiment.

The equal organization, shown on the right side of Figure 1 , is a case in which both the UC and the CC information lead to the same assessment of the causal strength of the items. The values of UC and CC for the two causes in this organization are all positive. For convenience, we label the cause with the higher $\mathrm{UC}$ and $\mathrm{CC}$ values the $\mathrm{su}$ perior cause and the cause with the lower $\mathrm{UC}$ and $\mathrm{CC}$ values the alternative cause.

The Simpson's paradox organization, shown on the left side of Figure 1, is a case in which the CC information yields a qualitatively different assessment of the causal strength of one of the causes than does the UC information. As is shown in Figure 1, the more powerful superior cause has both a positive UC and a positive CC. In contrast, the less powerful alternative cause has a positive UC but actually has a negative CC.

The logic of our study is straightforward. We exposed participants to a manipulation of self-construal, giving them either a relatively independent or a relatively interdependent self-construal. Then, we exposed them to events for the two contingency organizations shown in Figure 1. The expected results for the Simpson's paradox organization depended on the degree to which the people attended to contextual information and, hence, used CC, rather than $\mathrm{UC}$, information. The participants given an independent self-construal, who should be relatively insensitive to contextual information, should decide that both the superior cause and the alternative cause promote the effect. In contrast, the participants given an interdependent self-construal should decide that the superior cause promotes the effect but that the alternative cause inhibits the effect. When shown the equal organization, the participants should decide that both the superior cause and the alternative cause promote the effect. Furthermore, the participants should conditionalize overall and rate the alternative cause in the equal organization as more effective than the alternative cause in the Simpson's paradox organization.

\section{METHOD}

\section{Participants}

Sixty undergraduate students at the University of Texas at Austin were given course credit for their participation. They were randomly assigned to the interdependent-primed (henceforth, INT), independent-primed (henceforth, IND), or control condition. ${ }^{2}$

\section{Design}

The experiment used a 3 (self-construal: independent, interdependent, or control) $\times 2$ (organization type: Simpson's paradox or equal) design. Self-construal was manipulated between subjects. Organization type was within subjects.

\section{Materials and Procedure}

Self-construal manipulation. To manipulate the participants' self-construal, we followed Gardner et al. (1999). The participants in the INT and IND conditions were given a story describing a trip. The two versions differed only with respect to whether the pronouns were independent (e.g., I and mine) or interdependent (e.g., we and ours). The participants' task was to circle all the pronouns in the paragraph. The participants in the control condition were not given a prime. After completing the word search, all the participants completed a self-construal task (the twenty statements task, or TST; Kuhn \& McPartland, 1954). ${ }^{3}$ In this task, the participants were asked to provide 20 self-descriptions. Responses were then coded as independent if they described a personal attribute (trait, ability, physical descriptor, or attitude; e.g. " "I am intelligent") and as interdependent if they described a role in an important relationship (e.g., "I am engaged to marry Ben") or membership in a social group (e.g., "I am a student at the University of Texas). ${ }^{4}$ To ensure that the differences between conditions were not due to mood factors, we also measured the participants' positive/negative mood with the PANAS (Positive and Negative Affect Schedule) (Watson, Clark, \& Tellegen, 1988).

Contingency organizations. To assess the participants' relative sensitivities to CC, we created two contingency organizations (Figure 1), following Spellman et al. (2001). As was discussed in the introduction, in the Simpson's paradox and equal organizations, there were two causes: superior and alternative. In the Simpson's paradox organization, the alternative cause appears to aid in the blooming of the flower when considered alone $(\mathrm{UC}=.20)$ but actually inhibits flower growth when conditionalized on the superior cause $(\mathrm{CC}=$ -.13 ). In the equal organization, the alternative cause is less effective than the superior cause, as in the Simpson's paradox organization, but it clearly aids blooming; both the CC and the UC are .20.

The two superior causes in the Simpson's paradox and equal organizations have a UC of .60 and CCs of .67 and .60, respectively, indicating that they are stronger causes than the alternative cause in each organization.

Cover story. Following Spellman et al. (2001), we presented a scenario in which flowers bloomed or did not bloom after the use or nonuse of two liquids that were introduced as possible fertilizers. We used the same methods as Spellman et al. (2001) for the learning phase and the test phase.

Learning phase (trial-by-trial presentation). After reading the cover story, on each trial, the participants saw a picture of a plant and two different-colored liquids (blue, red, green, or yellow). In the picture, both liquids were poured (used), one was poured and the other was not (not used), or neither was poured. At the bottom of the screen was the question, "Do you think the plant will produce a flower?" The participants were given as much time as they needed to respond with a yes or no keypress. The participants were then given feedback for $5 \mathrm{sec}$ on the next screen, where the same plant was shown either blooming or not blooming.

Each participant completed four blocks of trials (two blocks for the Simpson's paradox organization and two blocks for the equal organization). Assignment of colors to each organization was counterbalanced across participants, and the order of block presentation was randomized. In each block, a blocked randomization technique was used for presenting the trial-by-trial information, so that each of five sections contained $20 \%$ of the information from each cell given in Figure 1, with order of presentation randomized within each section, yielding a total of 40 trials across sections. After each block, they were instructed to ignore events, outcomes, and colors in the previous blocks for their evaluation of the two causes in the current block.

Test phase (efficacy and confidence ratings). After each block, the participants were asked to judge the efficacy of each cause on a scale from -100 to 100 , in which negative numbers indicated that the liquid was a flower growth inhibitor, zero meant that it had no effect, and positive numbers meant that it was a flower growth fertilizer. Then they were asked to make confidence ratings on a scale from 0 (no confidence) to 10 (complete confidence) that the judgment was correct. The data were analyzed by averaging across the two replications of each type of organization. 


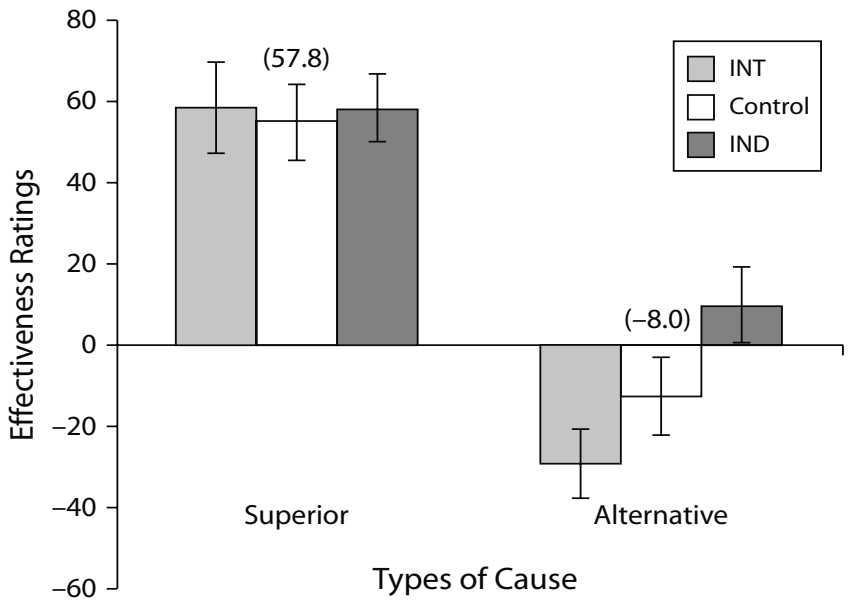

Figure 2. Participants' responses for the superior and alternative causes in the interdependent (INT), control, and independent (IND) conditions for the Simpson's paradox organization. For alternative causes, all the conditions were different (all comparisons at .05). The numbers in parentheses for the control group are the results in Spellman, Price, and Logan's (2001, Experiment 2) study. Error bars represent standard errors.

\section{RESULTS}

We first checked the effectiveness of our self-construal manipulation, using the participants' scores on the TST scale (intercoder reliability $=.92$ ). As was expected, the TST score was higher for the participants in the INT condition $(M=.32)$ than for those in the IND condition $(M=$ $.11)$, with the participants in the control condition $(M=$ .22 ) intermediate between the two. Planned $t$ tests revealed significant differences among all groups at the .05 level.

There were no significant differences in confidence ratings, the level of positive/negative mood, or participants' trial-by-trial responses for individual combinations of presence/absence of causes (i.e., individual cells on the contingency tables in Figure 1) between conditions (in all cases, $p>$.4). Furthermore, there was no effect of order of block presentation or effect of color assignment (in all cases, $p>.5)$.

\section{Tests of Conditionalization}

Figures 2 and 3 illustrate the participants' ratings for the causes in the two organizations. Our primary predictions focus on the ratings given to the two alternative causes in the Simpson's paradox and the equal organizations. Before examining these predictions, however, we examined the participants' ratings for the two superior causes in each organization to ensure that people were sensitive to the overall contingency structure (see Figures 2 and 3). Of importance, people judged these superior causes to strongly promote the effect (all ratings were greater than 50). There were no significant differences in the ratings for these two causes across conditions (in all cases, $p>.5$ ).

To test for conditionalization, we examined two indexes of performance. First, we examined the ratings for the alternative cause in the Simpson's paradox organization (see
Figure 2) and in the equal organization (see Figure 3). In the Simpson's paradox organization, this cause had a positive UC and a negative CC. Thus, greater attention to context would lead to relatively more negative ratings of causal efficacy. Consistent with this proposal, people primed with an independent self-construal gave higher ratings to the alternative cause $(M=10.0)$ than did people primed with an interdependent self-construal $(M=-29.1)[t(38)=5.33$, $p<.01]$. The participants in the control condition gave intermediate ratings $(M=-12.7)$, which differed significantly from those for the INT and IND groups.

In the equal organization, people primed with interdependence gave lower ratings to the alternative cause $(M=$ 4.4) than did people primed with independence $(M=20.6)$ $[t(38)=2.31, p<.05$; see Figure 3]. The control group ratings $(M=20.4)$ were significantly different from those for the INT group. We will discuss possible explanations for the group differences in the Discussion section.

In all the groups, people's ratings for the alternative cause in the Simpson's paradox organization were significantly lower than those for the alternative cause in the equal organization (for all comparisons, $p<.05$ ).

Next, we examined whether the differences in effectiveness ratings between groups for the alternative cause in the Simpson's paradox and equal organizations were mediated by the scale values on the TST. A Sobel (1982) test indicated that the TST, as a mediator, was indeed responsible for the difference in effectiveness ratings for alternative cause between the three conditions in the Simpson's paradox organization (Sobel test $=2.58, p<$ .01 ) and in the equal organization (Sobel test $=2.25, p<$ $.05)$. Furthermore, the relationship between the ratings for the alternative cause and the scale values on the TST was negative $(r=-.44, p<.01$, and $r=-.39, p<.01$,

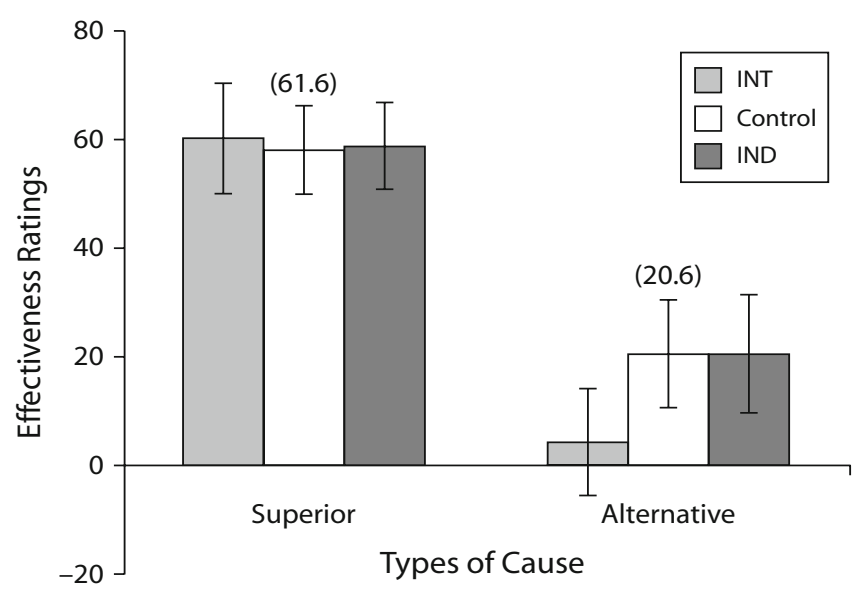

Figure 3. Participants' responses for the superior and alternative causes in the interdependent (INT), control, and independent (IND) conditions for the equal organization. For alternative causes, the INT condition showed lower ratings than did the other two, which were not different from each other (all comparisons at .05). The numbers in parentheses for the control group are the results in Spellman, Price, and Logan's (2001, Experiment 2) study. Error bars represent standard errors. 
respectively), meaning that relatively greater interdependence was associated with relatively smaller judgments of causal efficacy for the alternative cause, or relatively greater conditionalization.

\section{DISCUSSION}

This study extends previous research suggesting that individuals with a relatively greater interdependent selfconstrual are more sensitive to contextual information than are those with a more independent self-construal. We demonstrated this finding in the domain of causal induction. Relative to those with an independent self-construal, those with an interdependent self-construal showed a greater propensity to conditionalize their judgments on an alternative cause.

Our study followed the design in Spellman et al.'s (2001) work on conditionalization. Indeed, the results of our control condition (in which self-construal was not manipulated) provide a replication of their overall finding that judgments of the causal efficacy of the alternative cause were lower in the Simpson's paradox organization than in the equal organization.

We also found that the participants in the interdependent condition gave lower ratings of the causal efficacy of the alternative cause in the equal organization than might be expected given the actual contingency. Although not the central focus of this study, this difference may reflect discounting. Discounting occurs when the judged causal strength of a cue is lower than expected because it has been learned in the presence of a stronger cue (e.g., Price \& Yates, 1993; Tangen \& Allan, 2003). ${ }^{5}$ Further research will address this possibility by using the method developed by Goedert and Spellman (Goedert, Harsch, \& Spellman, 2005; Goedert \& Spellman, 2005) that was specifically designed to address the role of discounting in causal judgment.

We believe that our results could be misinterpreted in two key ways. First, our group differences should not be taken as evidence that all the interdependent participants used only CCs and all the independent participants used only UCs. We have demonstrated that being more interdependent makes conditionalization more likely. We calculated the number of participants that we believed had used the $\mathrm{UC}$, rather than the CC, by classifying the participants on the basis of two criteria: (1) They gave a positive rating to the alternative cause in the Simpson's paradox organization, and (2) the absolute value of the difference in their average ratings for the alternative causes in the Simpson's paradox and the equal organizations was not greater than 10 (since both had identical UCs in their respective organizations). Of the people primed with an independent self-construal, $35 \%$ were classified as using UC, as compared with only $5 \%$ of the people primed with interdependence $\left[\chi^{2}(1)=\right.$ $5.63, p<.05] .{ }^{6}$ Of the people in the control condition, $10 \%$ were classified as using $\mathrm{UC}$, which was significantly different from the independent condition.

Second, a possible conclusion from the present study is that people with an interdependent self-construal are more accurate in their causal reasoning than are those with an independent self-construal. By extension, this conclusion might suggest that members of cultures in which interdependence is more prominent (e.g., East Asian cultures) are more accurate in their causal judgments than are members of cultures in which independence is more prominent (e.g., Western cultures). We believe that this conclusion is not warranted. In our study, both the interdependent and the independent groups showed biases in their causal judgments, but the biases were in different directions. For example, the independent group gave causal efficacy judgments to the alternative cause in the Simpson's paradox organization that were much higher than the actual conditional contingency, but the interdependent group gave causal efficacy judgments that were too low. In contrast, the control condition showed relatively unbiased judgments. However, of note, in the equal organization, the interdependent group did exhibit bias, whereas the independent group did not.

Our findings have implications both for research on individual differences and for work on causal reasoning. On the side of individual differences, in many previous studies, tasks have been explored for which it is difficult to characterize exactly what the participants are doing. For example, Masuda and Nisbett (2001) found that recognition memory for objects was more strongly influenced by the background against which the objects were initially presented for East Asians than for Westerners. This finding could reflect differences in attention to the background by the members of different cultures, or it could reflect the use of a different response criterion for stating that an object had been seen before by the members of each culture (see Kim \& Markman, 2006).

By using causal induction as a domain, we have a rigorous mathematical foundation for determining the degree to which people attend to context. This method parallels recent studies of motivational factors in learning that have fit mathematical models to classification tasks in order to assess the influence of regulatory fit on learning (Maddox, Baldwin, \& Markman, 2006; Markman, Baldwin, \& Maddox, 2005).

The present results are also interesting from the point of view of causal reasoning. Previous research has documented situations in which people use UC and CC. Research has not explored the personality factors that influence individuals to favor these sources of information.

Within the domain of causal reasoning, the present results raise some interesting questions. First, although self-construal influenced both conditionalization and, possibly, discounting, these two processes may be cognitively dissociable (Goedert et al., 2005). Thus, it is not clear that manipulations of self-construal will affect discounting in the same way that it affects conditionalization. In addition, there are many ways to model the effects of self-construal on causal judgment. One would be to assume that individuals calculate contingencies by using the formulas described above. On this view, individuals with an interdependent self-construal will be more likely than those with an independent self-construal to calculate CC information. A second explanation focuses on associative 
models (e.g., Rescorla \& Wagner, 1972). Recent formulations of associative models suggest that individuals may use contextual information to encode both the presence and the absence of particular cues (Markman, 1989; Van Hamme \& Wasserman, 1994). Those with an interdependent self-construal may be more likely than those with an independent self-construal to explicitly note the absence of a cue. Future research should be directed at these possibilities.

\section{AUTHOR NOTE}

This research was supported by NIDA Grant NIH 1 R21 DA01521101A1 to the third author. The authors thank Serge Blok, John Dennis, Jeff Laux, and Jon Rein for discussions about the study, as well as Rachel Bernard, Patrick Brown, Barbara Krei, Brian Gurbach, Andrew Friedman, Catlynn Phillips, and Leora Orent for help conducting the experiment. The authors also thank Bobbie Spellman, Mike Oaksford, and an anonymous reviewer for useful comments on a previous draft of the manuscript. Correspondence concerning this article should be addressed to K. Kim, Department of Psychology, Ajou University, Suwon, Korea 443-749 (e-mail: kyungilkim@ajou.ac.kr).

\section{REFERENCES}

Busemeyer, J. R., Myung, I. J., \& McDaniel, M. A. (1993). Cue competition effects: Empirical tests of adaptive network learning models. Psychological Science, 4, 190-195.

Cheng, P. W. (1993). Separating causal laws from causal facts: Pressing the limits of statistical relevance. In D. L. Medin (Ed.), The psychology of learning and motivation (Vol. 30, pp. 215-264). New York: Academic Press.

Choi, I., Nisbett, R. E., \& Norenzayan, A. (1999). Causal attribution across cultures: Variation and universality. Psychological Bulletin, 125, 47-63.

Constantine, M. G. (2001). Independent and interdependent selfconstruals as predictors of multicultural case conceptualization ability in counsellor trainees. Counselling Psychology Quarterly, 14, 33-42.

Cross, S. E., \& Madson, L. (1997). Models of the self: Self-construals and gender. Psychological Bulletin, 122, 5-37.

Gardner, W. L., Gabriel, S., \& Lee, A. Y. (1999). "I" value freedom, but "we" value relationships: Self-construal priming mirrors cultural differences in judgment. Psychological Science, 10, 321-326.

Goedert, K. M., Harsch, J., \& Spellman, B. A. (2005). Discounting and conditionalization: Dissociable cognitive processes in human causal inference. Psychological Science, 16, 590-595.

Goedert, K. M., \& Spellman, B. A. (2005). Nonnormative discounting: There is more to cue interaction effects than controlling for alternative causes. Learning \& Behavior, 33, 197-210.

Haberstroh, S., Oyserman, D., Schwarz, N., Kuhnen, U., \& Ji, L. J. (2002). Is the interdependent self more sensitive to question context than the independent self? Self-construal and the observation of conversational norms. Journal of Experimental Social Psychology, 38, 323-329.

Hong, Y. Y., \& CHIU, C. Y. (2001). Toward a paradigm shift: From crosscultural differences in social cognition to social-cognitive mediation of cultural differences. Social Cognition, 19, 181-196.

Ji, L. J., Peng, K. P., \& Nisbett, R. E. (2000). Culture, control, and perception of relationships in the environment. Journal of Personality \& Social Psychology, 78, 943-955.

Ji, L. J., ZhANG, Z. Y., \& NisBett, R. E. (2004). Is it culture or is it language? Examination of language effects in cross-cultural research on categorization. Journal of Personality \& Social Psychology, 87, 57-65.

Kim, K., \& Markman, A. B. (2006). Differences in fear of isolation as an explanation of cultural differences: Evidence from memory and reasoning. Journal of Experimental Social Psychology, 42, 350-364.

KuHN, M. H., \& McPartland, T. (1954). An empirical investigation of self-attitudes. American Sociological Review, 19, 69-76.

Kuhnen, U., Hannover, B., \& Schubert, B. (2001). The semanticprocedural interface model of the self: The role of self-knowledge for context-dependent versus context-independent modes of thinking. Journal of Personality \& Social Psychology, 80, 397-409.

Kunnen, U., \& Oyserman, D. (2002). Thinking about the self influences thinking in general: Cognitive consequences of salient selfconcept. Journal of Experimental Social Psychology, 38, 492-499.

Lopez, A., Atran, S., Coley, J. D., Medin, D. L., \& Smith, E. E. (1997). The tree of life: Universal and cultural features of folkbiological taxonomies and inductions. Cognitive Psychology, 32, 251-295.

Maddox, W. T., Baldwin, G. C., \& Markman, A. B. (2006). A test of the regulatory fit hypothesis in perceptual classification learning. Memory \& Cognition, 34, 1377-1397.

MARKMAN, A. B. (1989). LMS rules and the inverse base-rate effect: Comment on Gluck and Bower (1988). Journal of Experimental Psychology: General, 118, 417-421.

Markman, A. B., Baldwin, G. C., \& Maddox, W. T. (2005). The interaction of payoff structure and regulatory focus in classification. Psychological Science, 16, 852-855.

Markus, H. R., \& Kitayama, S. (1991). Culture and the self: Implications for cognition, emotion, and motivation. Psychological Review, 98, 224-253.

MASUdA, T., \& NisbetT, R. E. (2001). Attending holistically versus analytically: Comparing the context sensitivity of Japanese and Americans. Journal of Personality \& Social Psychology, 81, 922-934.

Melz, E. R., Cheng, P. W., Holyoak, K. J., \& Waldmann, M. R. (1993). Cue competition in human categorization: Contingency or the Rescorla-Wagner learning rule. Comment. Journal of Experimental Psychology: Learning, Memory, \& Cognition, 19, 1398-1410.

Peng, K. P., \& Nisbett, R. E. (1999). Culture, dialectics, and reasoning about contradiction. American Psychologist, 54, 741-754.

Price, P. C., \& Yates, J. F. (1993). Judgmental overshadowing: Further evidence of cue interaction in contingency judgment. Memory \& Cognition, 21, 561-572.

Proffitt, J. B., Coley, J. D., \& Medin, D. L. (2000). Expertise and category-based induction. Journal of Experimental Psychology: Learning, Memory, \& Cognition, 26, 811-828.

Rescorla, R. A., \& Wagner, A. R. (1972). A theory of Pavlovian conditioning: Variations in the effectiveness of reinforcement and nonreinforcement. In A. H. Black \& W. F. Prokasy (Eds.), Classical conditioning II: Current research and theory (pp. 64-69). New York: Appleton-Century-Crofts.

Sobel, M. E. (1982). Asymptotic intervals for indirect effects in structural equations models. In S. Leinhart (Ed.), Sociological methodology (pp. 290-312). San Francisco: Jossey-Bass.

Spellman, B. A. (1996a). Acting as intuitive scientists: Contingency judgments are made while controlling for alternative potential causes. Psychological Science, 7, 337-342.

Spellman, B. A. (1996b). Conditionalizing causality. In D. L. Medin (Ed.), The psychology of learning and motivation (Vol. 34, pp. 167206). San Diego: Academic Press.

Spellman, B. A., Price, C. M., \& Logan, J. M. (2001). How two causes are different from one: The use of (un)conditional information in Simpson's paradox. Memory \& Cognition, 29, 193-208.

Tangen, J. M., \& Allan, L. G. (2003). The relative effect of cue interaction. Quarterly Journal of Experimental Psychology, 56B, 279-300.

Trafimow, D., Triandis, H. C., \& Goto, S. G. (1991). Some tests of the distinction between the private self and the collective self. Journal of Personality \& Social Psychology, 60, 649-655.

van Baaren, R. B., Maddux, W. W., Chartrand, T. L., De Bouter, C., \& VAN KNIPPENBERG, A. (2003). It takes two to mimic: Behavioral consequences of self-construals. Journal of Personality \& Social Psychology, 84, 1093-1102.

Van Hamme, L. J., \& Wasseerman, E. A. (1994). Cue competition in causality judgments: The role of nonpresentation of compound stimulus elements. Learning \& Motivation, 25, 127-151.

Wasserman, E. A., Chatlosh, D. L., \& Neunaber, D. J. (1983). Perception of causal relations in humans: Factors affecting judgments of response outcome contingencies under free-operant procedures. Learning \& Motivation, 14, 406-432.

Watson, D., Clark, L. A., \& Tellegen, A. (1988). Development and validation of brief measures of positive and negative affect: The PANAS scales. Journal of Personality \& Social Psychology, 54, 1063-1070. 


\section{NOTES}

1. Spellman and her colleagues (Spellman, 1995a; Spellman et al., 2001) discriminated between the conditional $\Delta P$ for a candidate cause (A) with a second candidate cause (B) present $\left(\Delta P_{A \mid \sim B}\right)\left[\Delta P_{A \mid \sim B}=P(\mathrm{E} \mid \mathrm{A}\right.$ and $\mathrm{B})$ $-P(\mathrm{E} \mid \sim \mathrm{A}$ and $\mathrm{B})]$ and the conditional $\Delta P$ for $\mathrm{A}$ with $\mathrm{B}$ absent $\left(\Delta P_{A \mid \sim B}\right)$ $\left[\Delta P_{A \mid \sim B}=P(\mathrm{E} \mid \mathrm{A}\right.$ and $\sim \mathrm{B})-P(\mathrm{E} \mid \sim \mathrm{A}$ and $\left.\sim \mathrm{B})\right]$.

2 . There were about the same number of men and women in each condition $(9 / 11,10 / 10$, and 9/11 male/female in the INT, IND, and control conditions, respectively). No significant effects of gender were obtained on the TST check or dependent variables.

3. The TST was given right after the manipulation of self-construal, and so it is possible that the strength of our manipulations reflects an interaction between the pronoun-circling task and the TST. The degree to which the TST influences the manipulation of self-construal is an in- teresting question for future research but does not affect the conclusions drawn in this study.

4. Completions that referred to transient states (e.g., "I am hungry") were relatively rare (fewer than $4 \%$ ) and were not counted as self-definitions.

5. Discounting may occur to some degree in cases in which the $\mathrm{CC}$ of a cue is less than its UC. Discounting has also been observed simply given the presence of a strong alternative cause even when there is no reason to conditionalize on this alternative (Busemeyer, Myung, \& McDaniel, 1993).

6 . There was no significant difference in trial-by-trial responses between the UC user group and others.

(Manuscript received October 16, 2005; revision accepted for publication February 7, 2006.) 